EXTRACTA MATHEHATICAE $3, n .2,64-66$ (1988)

\title{
NONSELFADJOINT BOUNDARY VALUE PROBLEMS AT RESONANCE. NONLINEARITIES WHICH MAY GROW LINEARLY
}

\section{Arias}

Departamento de Matemática Aplicada. Universidad de Granada 18071. Granada. SPAIN.

1980 Mathematics Subject Classification, 35630.

Let $\Omega$ be a finite complete measure space and $H=L^{2}(\Omega)$ be the space of Lebesgue measurable square integrable functions on $\Omega$. We consider the existence of solutions of the equation

$$
L u-\lambda u=g(\cdot, u)+f,
$$

where $\mathrm{L}:$ dom $\mathrm{L} \subset \mathrm{H} \longrightarrow \mathrm{H}$ is a Fredholm linear operator with index zero and compact generalized right inverse, $K, \lambda$ is an eigenvalue of $L, f \in H$ and $g: \Omega \times \mathbb{R} \longrightarrow \mathbb{R}$ is a nonlinear fun ction satisfying Caratheodory conditions which grows at most linearly.

Recently, Iannacci and Nkashama, [3], have obtained an existence Theorem for (1) when $L$ is selfadjoint, $\lambda=\lambda_{n}$ is the $n$-th eigenvalue of $L$ and the nonlinear function $g$ lies between two consecutives eigenvalues and may be unbounded and reach the eigenvalue $\lambda_{n+1^{-} \lambda_{n}}$ on a subset of positive me asure.

When $L$ is nonselfadjoint, there exist few results about (1). Ahmad, [1] (see, also [2]) studies (1) when L is an uni formly elliptic linear operator of second order with Dirich let conditions on the boundary of a bounded open set $\Omega$ of $\mathbb{R}^{n}$, $\lambda=\lambda_{1}$ is the principal eigenvalue and $g(., u) \equiv g(u)$ is locally Lipchitzian on $\mathbb{R}$. He shows that there exists a constant $d_{0}>\lambda_{1}$ depending only on $L\left(d_{0}=\lambda_{2}\right.$ if $L$ is selfadjoint $)$ such that if $\lim \sup (g(u) / u)<d_{0}-\lambda_{1},($ and $f$ satisfies a Landesman La$|u| \rightarrow \infty$ zer type condition), (1) is solvable.

we present some abstract results for (1) valid for $L$ nonselfadjoint which generalize in certain cases those of $[1],[3]$.

Let $L$ be satisfying the previous conditions. It is not restrictive to assume $\lambda=0 \varepsilon \sigma(L)(=$ eigenvalues of $L$ ) and con sider the equation

$$
L u=g(., u)+f, \quad u \in d o m L .
$$


Suppose moreover that
(C1) For any $\phi$ Eker $L, \phi \neq 0$, there exists $\Psi \varepsilon k e r L^{*}-\{0\}$ with
$\phi(x) \Psi(x) \geq 0$ a.e. $\Omega$ and $|N(\phi)-N(\Psi)|=0$,
where $L^{*}$ is the adjoint operator of $L, N(f)=\{x \in \Omega \mid f(x)=0\}$ and 1.1 denotes the measure of $\Omega$.

THEOREM.- Let $d_{0}=\sup \{d \geq 0 /$ if $\mu \in H, O \leq \mu \leq d$ a.e. $\Omega$ and $\phi$ is solution of $L u=\mu u$, then $\phi \varepsilon k e r L$ \} (one can prove $d_{0}>0$ ). Suppose

(H1) There exist $\gamma, k, \alpha, \beta \in H, 0 \leq \gamma \leq d<d_{0}$ a.e. $\Omega$ such that $|g(x, u)| \leq \gamma(x)|u|+k(x), u \in R, g(x, u) \geq \alpha(x), u \geq 0, g(x, u) \leq \beta(x)$, $u \leq 0, \quad$ a.e. $x \in \Omega$.

(H2) For any $\psi \in k$ er $L^{*}-\{0\}, \int_{\Omega} f \psi>\int_{\Omega} \bar{g}(-\infty) \psi^{-}-\int_{\Omega} g(+\infty) \Psi^{+}$ where $\bar{g}(-\infty)(x)=1$ im sup $g(x, u), \underline{g}(+\infty)(x)=1$ im inf $g(x, u), \psi^{+}(x)=$

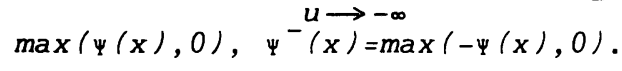

Then, equation (2) has at least one solution.

The proof of this Theorem is based in topological methods and it is essential to obtain apriori bounds. These aprio$r i$ bounds are obtained by using a contradiction argument and comparing with a linear problem.

The following Proposition sets up the relation between the previous Theorem and the results in [1], [3].

PROPOSITION.- Suppose, al so

(C2) $0<\lambda_{1}=\inf \{\operatorname{Re} \lambda>0 \mid \lambda \varepsilon \sigma(L)\} \varepsilon \sigma(L)$ and $\left\|\left(L-\frac{\lambda}{2} I\right)^{-1}\right\|_{H \rightarrow H}=\frac{2}{\lambda_{1}}$.

Then, $d_{0}=\lambda_{1}$ and we can replace the condition $0 \leq r \leq d<d_{0}$ in (H1) by $0 \leq \gamma<\lambda_{1}$, a.e. $\Omega$.

REMARK. - If $L$ is selfadjoint, conditions ( $C 1$ ) and ( $C 2$ ) are trivially satisfied (when $L$ has positive eigenvalues) and (H1) allows to the nonlinearity $g$ to lie between two consecutives eigenvalues of $L$.

\section{EXAMPLES.-}

Let $\Omega$ be a bounded open set of $\mathbb{R}^{n}, T>0, H=L^{2}((\mathbb{R} / T Z) \times \Omega)$ and $\lambda_{n}$ the $n-t h$ eigenvalue of Dirichlet problem for the Laplacian operator on $\Omega$ (we denote by $A$ ). Define 
$\mathrm{L}: \operatorname{dom} \mathrm{L} \subset \mathrm{H} \longrightarrow \mathrm{H}$ by

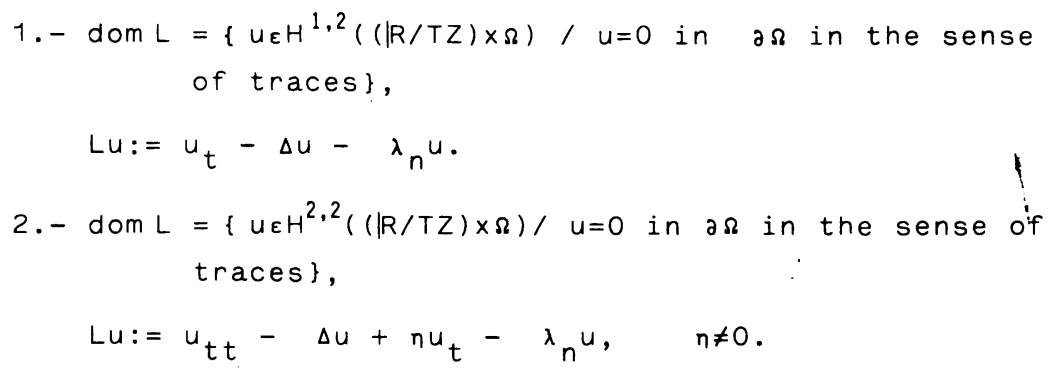

\section{REFERENCES}

[1] AHMAD, S., Nonlinear Analysis, V. 10, N.2, (1986), 147-156.

[2] CESARI, L., PUCCI, P., Nonlinear Analysis, V.9, N.11, (1985), 1227-1241.

[3] IANNACCI, R., NKASHAMA, M.N., Nonlinear Analysis, V.11, n.4, (1987), 445-473. 